\title{
ORIGINAL ARTICLE \\ Multigenerational hybridisation and its consequences for maternal effects in Atlantic salmon
}

\begin{abstract}
PV Debes ${ }^{1}$, DJ Fraser ${ }^{2}$, MC McBride ${ }^{1}$ and JA Hutchings ${ }^{1,3}$
Outbreeding between segregating populations can be important from an evolutionary, conservation and economical-agricultural perspective. Whether and how outbreeding influences maternal effects in wild populations has rarely been studied, despite both the prominent maternal influence on early offspring survival and the known presence of fitness effects resulting from outbreeding in many taxa. We studied several traits during the yolk-feeding stage in multigenerational crosses between a wild and a domesticated Atlantic salmon (Salmo salar) population up to their third-generation hybrid in a common laboratory environment. Using cross-means analysis, we inferred that maternal additive outbreeding effects underlie most offspring traits but that yolk mass also underlies maternal dominant effects. As a consequence of the interplay between additive and dominant maternally controlled traits, offspring from first-generation hybrid mothers expressed an excessive proportion of residual yolk mass, relative to total mass, at the time of first feeding. Their residual yolk mass was $23-97 \%$ greater than those of other crosses and $31 \%$ more than that predicted by a purely additive model. Offspring additive, epistatic and epistatic offspring-bymaternal outbreeding effects appeared to further modify this largely maternally controlled cross-means pattern, resulting in an increase in offspring size with the percentage of domesticated alleles. Fitness implications remain elusive because of unknown phenotype-by-environment interactions. However, these results suggest how mechanistically co-adapted genetic maternal control on early offspring development can be disrupted by the effects of combining alleles from divergent populations. Complex outbreeding effects at both the maternal and offspring levels make the prediction of hybrid phenotypes difficult.

Heredity (2013) 111, 238-247; doi:10.1038/hdy.2013.43; published online 8 May 2013
\end{abstract}

Keywords: cross-means analysis; outbreeding depression; heterosis; intraspecific hybridisation; maternal effects

\section{INTRODUCTION}

Understanding the genetic architecture of population divergence allows for the prediction of generational trajectories of hybrids by their phenotype and relative fitness, both of which can influence adaptation, speciation and conservation- or economical-agricultural breeding strategies (Lynch, 1991; Burke and Arnold, 2001; Sørensen et al., 2008). A particular genetic architecture, such as the presence or interplay of dominance (interaction of alleles at the same locus) and epistasis (interaction of alleles at different loci), governs the genotypedependent trajectory of the phenotype and the mechanisms of hybrid fitness across generations (Lynch, 1991).

Predicting the effects of outbreeding can be further complicated by maternal effects, defined as the maternal contribution to the offspring phenotype that can underlie both environmental and genetic effects (Räsänen and Kruuk, 2007; Wolf and Wade, 2009). Maternal effects act through maternal provisioning to offspring other than that generated by meiotic or cytoplasmic-derived genetic parental contribution (reviewed by Wolf and Wade, 2009). Maternal effects can, at least temporarily, outweigh or interact with the offspring genotype in forming a particular phenotype (Wolf, 2000). Hence, a major challenge is the disentanglement of maternal effects from environmental and direct offspring genetic effects (Willham, 1980; Kruuk and Hadfield, 2007).

In most studies on wild populations, maternal effects are not assessed for their genetic architecture (Räsänen and Kruuk, 2007), although genetic-based maternal effects are of evolutionary and ecological importance, given their role as heritable modifiers of the development and phenotype of the offspring (Mousseau and Fox, 1998; Wolf and Wade, 2009). Maternal effects are indirect genetic effects that are founded in an individual other than the one measured (Wolf et al., 1998), which might be the reason for a lack of acknowledgment that maternal effects can also be affected by outbreeding. As such, genetic maternal effects might often remain undetected unless several generations are studied (Willham, 1980). Furthermore, the effect of outbreeding on maternal effects, i.e., maternal outbreeding effects, can only be studied by using hybrid dams.

Fishes of the family Salmonidae (including whitefish, trout, salmon) are suitable vertebrate study organisms for conducting studies of genetic divergence and genetic-based maternal effects. Most salmonid species occur as discrete populations isolated by strong philopatry, creating the potential for genetic differences through genetic drift and local adaptation (Fraser et al., 2011), and maternal effects are prevalent in this family, mostly related to egg and nest quality (Green, 2008). Furthermore, this fish family is affected by a rapidly growing aquaculture industry, in addition to other anthropogenic translocations such as stocking, all of which can lead to population interbreeding and conservation-related concerns associated with outbreeding depression (Utter and Epifanio, 2002).

${ }^{1}$ Department of Biology, Dalhousie University, Halifax, Canada; ${ }^{2}$ Department of Biology, Concordia University, Montreal, Canada and ${ }^{3}$ Centre for Ecology and Evolutionary Synthesis, Department of Biosciences, University of Oslo, Oslo, Norway

Correspondence: PV Debes, Department of Biology, Dalhousie University, 1355 Oxford Road, Halifax, B3H 4J1, Canada.

E-mail: paul.debes@dal.ca

Received 16 October 2012; revised 22 March 2013; accepted 29 March 2013; published online 8 May 2013 
Salmonid females typically release several thousand eggs, which can be divided experimentally and fertilised by multiple males, allowing for the study of outbreeding and population-specific maternal effects while controlling for individual maternal effects.

Although some studies have investigated the effects of outbreeding in early life stages of salmonids, most of these accounted only for individual maternal effects. We are aware of only two studies in which maternal between-population effects have been examined while simultaneously accounting for individual maternal variation (Houde et al., 2011; Aykanat et al., 2012). Most others, however, have ignored maternal effects by generating crosses in a non-reciprocal fashion or averaging reciprocal cross data, probably because of the logistic challenges associated with undertaking multigenerational studies. We are unaware of any study of maternal outbreeding effects in wild vertebrate populations.

We investigated several maternally influenced, fitness-related traits and their response to outbreeding in Atlantic salmon (Salmo salar) during the yolk-feeding period encompassing hatch and time of first feeding, both of which are major events in the early life of fishes. Many salmonids bury their eggs in river gravel where eggs develop from which alevins hatch while relying on maternally provided egg yolk as the major source of energy and nutrients (Kamler, 1992) until they emerge from the gravel and begin external feeding as fry.

We created reciprocal crosses between an endangered wild population and its major local domesticated counterpart up to their third hybrid generation. To minimise environmental maternal and environmental offspring effects, crosses were maintained in a common laboratory for three generations. We then analysed traits from 14 reciprocal crosses by using a cross-means analysis within a mixed model framework. In particular, we investigated the effect of outbreeding on maternal body size and egg size, and on offspring survival, hatch time, yolk size and body size at both hatch and time of first feeding. Our study emphasises the potential importance of additive and non-additive maternal outbreeding effects in early life, by quantifying maternal, offspring and epistatic offspring-by-maternal outbreeding effects for offspring trait means.

\section{MATERIALS AND METHODS}

\section{Study populations}

Outbreeding effects were studied in crosses between endangered wild Atlantic salmon (WW) from the Stewiacke River (Nova Scotia, Canada) and domesticated salmon (DD) derived from the Saint John River population (New Brunswick, Canada). Both founder populations were provided by the Department of Fisheries and Oceans (DFO). WW salmon were caught as juveniles in the river. DD salmon were derived from an aquaculture population founded by $50-100$ wild-caught individuals that underwent three generations of selection, primarily for rapid growth (Glebe, 1998). The two wild river populations are naturally separated by $\sim 200 \mathrm{~km}$ (waterway distance) and are divergent for neutral genetic and ecological parameters (reviewed by Fraser et al., 2010).

Gametes from the experimental founder generation were crossed in 2001, creating 10 reciprocal first-generation hybrid (F1 hybrids) full-sib families (using five dams and five sires from each population) and 10 full-sib families for each population. All 30 full-sib families were raised under common laboratory conditions at Dalhousie University, Halifax, Canada (details in Lawlor et al., 2008). In 2005, gametes from the 2001 generation were crossed to re-create parental populations and reciprocal F1 hybrids and to create secondgeneration hybrids (F2 hybrids $=\mathrm{F} 1 \times \mathrm{F} 1$; details in Fraser et al., 2010). Because only several WW families were available in 2005, a few additional WW fish from DFO were used to supplement the existing 2001 generation breeders. This might have caused a higher genetic diversity in F1 crosses than in F2 crosses of the 2005 generation. The 2005 generation was again raised under common environmental conditions (same ad libitum feeding regime, laboratory, water source, temperature, oxygen saturation, tank type, fish density, light intensity and regime) at Dalhousie University. In 2009, gametes from four crosses (WW, DD, F1 and F2 hybrids) of the 2005 generation were used to create 14 reciprocal crosses (the 2009 generation; Table 1, Figure 1).

For each generation, all potential parents were tagged, fin clipped and genotyped at three to six polymorphic microsatellite loci. This allowed for the assignment of offspring to their parents by exclusion principles to avoid the crossing of relatives to the level of second cousins, what will be termed crossing 'unrelated' parents.

\section{Breeding protocol and laboratory environment}

For the 2009 generation, eggs from a given dam were used to create all crosses possible according to her cross (Figure 1). All 14 crosses were created in equal family numbers during each of 5 days (Figure 2). Each of 64 randomly selected dams was crossed to one or two randomly selected yet unrelated sires (out of a

Table 1 Sample sizes for each of the 14 crosses of the 2009 Atlantic salmon generation for initially used dams, sires and created families and used sample sizes for each of the three developmental offspring stages for individuals, families (in parentheses) and number of dams and sires

\begin{tabular}{|c|c|c|c|c|c|c|c|c|}
\hline $\begin{array}{l}\text { Cross } \\
(q \times \delta)^{a}\end{array}$ & $\begin{array}{c}\text { Initial } \\
\text { dams } \times \text { sires }\end{array}$ & $\begin{array}{l}\text { Initial } \\
\text { families }\end{array}$ & $\begin{array}{c}\text { Egg individuals } \\
\text { (families) }\end{array}$ & $\begin{array}{c}\text { Egg } \\
\text { dams } \times \text { sires }\end{array}$ & $\begin{array}{c}\text { Alevin individuals } \\
\text { (families) }\end{array}$ & $\begin{array}{c}\text { Alevin } \\
\text { dams } \times \text { sires }\end{array}$ & $\begin{array}{l}\text { Fry individuals } \\
\text { (families) }\end{array}$ & $\begin{array}{c}\text { Fry } \\
\text { dams } \times \text { sires }\end{array}$ \\
\hline$W W \times W W$ & $15 \times 17$ & 32 & $179(22)$ & $11 \times 13$ & $151(21)$ & $11 \times 13$ & $176(22)$ & $11 \times 13$ \\
\hline $\mathrm{WW} \times \mathrm{F} 1$ & $15 \times 13$ & 21 & $126(15)$ & $10 \times 11$ & $112(15)$ & $10 \times 11$ & $118(15)$ & $10 \times 11$ \\
\hline$W W \times F 2$ & $15 \times 15$ & 21 & $128(15)$ & $11 \times 11$ & 109 (15) & $11 \times 11$ & $118(15)$ & $11 \times 11$ \\
\hline $\mathrm{F} 1 \times \mathrm{WW}$ & $15 \times 13$ & 20 & $133(16)$ & $12 \times 12$ & $113(15)$ & $12 \times 12$ & $125(16)$ & $12 \times 12$ \\
\hline $\mathrm{F} 2 \times \mathrm{WW}$ & $16 \times 15$ & 20 & $110(14)$ & $11 \times 11$ & $105(14)$ & $11 \times 11$ & $112(14)$ & $11 \times 11$ \\
\hline$W W \times D D$ & $15 \times 13$ & 23 & $143(17)$ & $11 \times 11$ & $116(16)$ & $11 \times 11$ & 135 (17) & $11 \times 11$ \\
\hline $\mathrm{F} 1 \times \mathrm{F} 1$ & $15 \times 17$ & 30 & $184(24)$ & $12 \times 17$ & $158(22)$ & $11 \times 15$ & $176(24)$ & $11 \times 15$ \\
\hline $\mathrm{F} 2 \times \mathrm{F} 2$ & $16 \times 15$ & 32 & 211 (24) & $12 \times 12$ & $183(24)$ & $12 \times 12$ & $192(24)$ & $12 \times 12$ \\
\hline $\mathrm{DD} \times \mathrm{WW}$ & $16 \times 14$ & 23 & $32(5)$ & $4 \times 5$ & $23(4)$ & $4 \times 4$ & $31(5)$ & $4 \times 4$ \\
\hline $\mathrm{F} 1 \times \mathrm{DD}$ & $15 \times 12$ & 19 & $112(14)$ & $12 \times 9$ & $102(13)$ & $11 \times 9$ & 109 (14) & $11 \times 9$ \\
\hline $\mathrm{F} 2 \times \mathrm{DD}$ & $16 \times 14$ & 20 & $115(14)$ & $11 \times 10$ & $106(14)$ & $11 \times 10$ & $111(14)$ & $11 \times 10$ \\
\hline $\mathrm{DD} \times \mathrm{F} 1$ & $16 \times 13$ & 22 & $12(2)$ & $2 \times 2$ & $11(2)$ & $2 \times 2$ & $16(2)$ & $2 \times 2$ \\
\hline $\mathrm{DD} \times \mathrm{F} 2$ & $16 \times 16$ & 22 & $12(2)$ & $2 \times 2$ & $11(2)$ & $2 \times 2$ & $16(2)$ & $2 \times 2$ \\
\hline $\mathrm{DD} \times \mathrm{DD}$ & $16 \times 17$ & 32 & $59(7)$ & $4 \times 6$ & $39(7)$ & $4 \times 6$ & $50(7)$ & $4 \times 6$ \\
\hline Total & $64 \times 77$ & 351 & $1556(191)$ & $39 \times 67$ & 1339 (184) & $39 \times 67$ & $1485(191)$ & $39 \times 67$ \\
\hline
\end{tabular}

aCross abbreviations are wild, WW; domesticated, DD; reciprocal first-generation hybrid, F1; and second-generation hybrid, F2. 


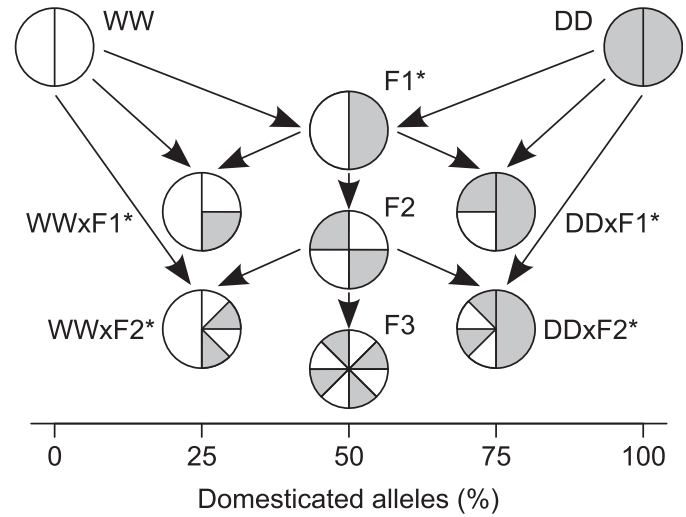

Figure 1 Schematic crossing design among four parental crosses of Atlantic salmon (labels beside symbols: wild, WW; domesticated, DD; first-generation hybrid, F1; second-generation hybrid, F2) that were used to create nine crosses of which five (marked by asterisks) were created in a reciprocal fashion, totalling 14 reciprocal crosses. The colours of the symbol pie charts represent percentage of alleles from WW (white) and DD (grey) and the extent of break-up of each vertically divided chart indicates the extend of relative genetic recombination between both populations.

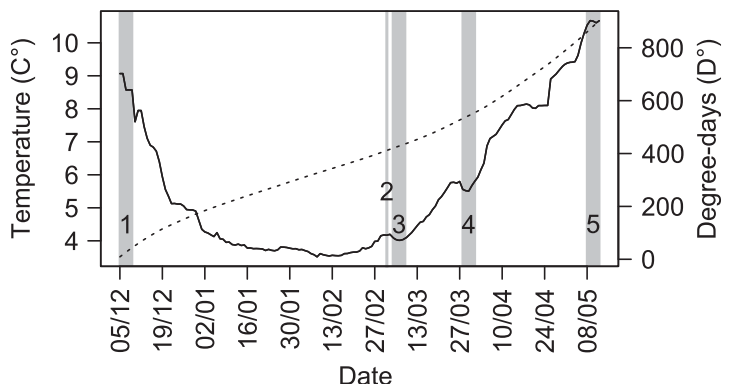

Figure 2 Average daily temperature (solid line) and cumulative degree-days (dotted line) for the duration of the experiment and for all the families of 14 crosses of the 2009 generation between wild and domesticated Atlantic salmon. Dates for events between December 2009 and May 2010 are indicated by vertical, grey bars across the plot area with 1 , fertilisation; 2 , shocking of eggs; 3 , sampling of eyed eggs; 4 , hatch and sampling of alevins; 5 , time of first feeding and sampling of fry.

total of 77 sires) from different crosses and to two randomly selected and unrelated sires from the same cross. Crossing was accomplished by dividing stripped eggs by volume into four to eight batches of $\sim 250$ eggs each into polystyrene foam bowls followed by fertilisation. The fertilised eggs were immediately placed family-by-family into one of 354 compartments $\left(13.8 \times 17.0 \mathrm{~cm}^{2}\right)$. Two compartments formed one plastic container, separated by fine mesh, and each compartment had mesh-covered holes $(3.8 \mathrm{~cm}$ diameter) on each side to permit water flow. In total, 177 plastic containers were put in groups of three into one of 59 similar 601 round tanks.

Each tank received dechlorinated, aerated municipal water at ambient temperature by a constant flow-through system. The latter was achieved by a spray bar to induce a circular-directed water flow. Each tank was equipped with a central, circular air stone to ensure sufficient oxygen supply and withintank temperature homogeneity. All compartments, plastic containers and tanks were established, using the same equipment and adjustments to minimise environmental among-family variability. Tanks, suspended at two levels, were known from previous years to exhibit small but systematic daily watertemperature differences (average maximum daily difference $0.2^{\circ}$ ), with upper level and tanks furthest from the supply being warmest. To prevent a temperature-by-cross bias, families were distributed in a stratified randomised fashion with equal proportions of families from each cross randomly distributed across each level. Temperature was measured daily for every tank $\left( \pm 0.1^{\circ}\right.$; range $3.5-10.8^{\circ}$; Figure 2 ), allowing cumulative degree-days $\left(D^{\circ}\right)$ to be calculated for each family. No replication at the family level was conducted due to tank-space limitations. Eggs were maintained in the dark until the termination of the experimental work.

\section{Sampling of traits}

During spawning, fork length $( \pm 1 \mathrm{~cm})$ and body wet mass $( \pm 5 \mathrm{~g})$ was recorded for each potential breeder. Initial numbers of fertilised eggs were counted, using photographs of each family compartment. Throughout the yolk-feeding period, opaque-turning dead eggs and dead alevins were manually removed approximately every second day to minimise the probability of fungal infection. At the time of first feeding, photographs were again taken from all families and survivors counted.

At an overall average $412 \mathrm{D}^{\circ}$, eggs from each family were physically stressed by heavy shaking in a bucket ('shocking'), which allowed for the identification of dead eggs. During shocking, compartment bottoms were fitted with artificial turf to minimise energy loss due to alevin movement (Marr, 1963). Shortly after shocking (Figure 2), 8-10 eyed eggs from each family were sampled consecutively during 5 days in the same order as spawned, fixed in buffered $10 \%$ formalin for $24 \mathrm{~h}$ and then preserved in phosphate-buffered saline with 0.1 sodium azide until further analyses.

Sampling of offspring individuals for trait measurements occurred at two stages during the yolk-feeding period (Figure 2): larvae at 50\% hatch (alevins) and unfed fry at the time of first feeding (fry). After hatch commenced, the percentage of hatched alevins was estimated daily by eye for each family and, when exceeding 50\%, eight (or less if unavailable) alevins were sampled, fixed and preserved as described previously. When families spawned on the same day had reached 100\% development, based on Kane (1988), eight (or less if unavailable) unfed fry were sampled from each family, fixed and preserved as described previously.

For preserved alevin and fry, individual standard length $( \pm 1 \mathrm{~mm})$ was recorded. The entire formalin-hardened yolk-sacs (including yolk-sac skin and oil) were precisely dissected from alevin and fry bodies and kept in individual pairs of yolk-sac and body, allowing for their separate dry mass measurements. Individual samples (including eyed eggs) were oven-dried at $60^{\circ}$ until no change in mass was noted in 24 -h intervals and dry mass was determined $( \pm 0.1 \mathrm{mg})$.

\section{Statistical analyses of maternal traits}

Differences in average fork length, body mass and (eyed) egg size among the four dam crosses (WW, DD, F1 and F2 hybrids) were examined. Length and mass (both Ln-transformed) were each assessed using a linear model with dam cross as a fixed term and common Gaussian distributed residuals. Dry mass of eyed eggs (egg size) was assessed using a linear mixed model (LMM) with dam cross as a fixed term, the direct product of dam identification ('dam') with dam cross (allowing different among-dam variances for dam crosses) and family identification ('family') as random terms and allowing independent strata of Gaussian distributed residuals among dam crosses. Correlations between dam traits (egg size vs Ln fork length and Ln body mass) were tested using Pearson's product moment correlation.

\section{Statistical analyses of offspring traits}

Survival and incubation period. Survival between fertilisation and time of first feeding was analysed based on the (logit-transformed) proportion of individuals surviving to the fry stage out of the initial number of eggs for each family. A LMM was used with cross as a fixed term, dam as a random term and allowing independent strata of Gaussian distributed residuals among reciprocal offspring crosses.

Average cumulative degree-days at $50 \%$ hatch (incubation period) of families was analysed using a LMM with cross as a fixed term, dam and tank identification ('tank') as random terms and a common Gaussian residual distribution. In this model, final number of live individuals per family ('density'), average eyed egg dry mass per family ('egg mass') and the product of the two ('biomass') were tested for their influence on incubation period by 
including them as fixed continuous covariates. These and all other continuous covariates, were centred by dividing each value by the sampling-period-specific mean. These covariates were tested because they might correlate negatively with water oxygen saturation (density, biomass), or positively with total oxygen egg demand (egg mass), and both might influence hatch or development.

Cross-means analyses of alevin and fry traits. For analyses, body length and dry mass of body and yolk for both alevins and fry were treated as six different traits to allow for testing of the main genetic architecture of each trait at different times by cross means analysis. Cross means and genetic outbreeding effects were estimated for each trait, using LMMs taking into account environmental effects and kinship among individuals. The analysis followed the general LMM:

$$
y=X_{\tau}+Z_{1} u_{1}+Z_{2} u_{2}+e
$$

where $y$ is a $n \times 1$ vector of individual observations of a given trait, $\tau$ is a $p \times 1$ vector of fixed continuous and/or categorical effects, $u_{1}$ is a $q \times 1$ vector of random effects assumed to be independent and Gaussian distributed with an overall mean of zero, $u_{2}$ is a $r \times 1$ vector of random animal effects correlated based on the additive relationship matrix (see below) and $e$ is a $n \times 1$ vector of Gaussian distributed residual errors with an overall mean of zero. $X, Z_{1}$ and $Z_{2}$ are incidence matrices relating observations $y$ to respective effects.

Temperature and oxygen saturation are known to influence development of yolk-feeding salmon (Kamler, 1992). Before assessing outbreeding effects, the influence of both environmental factors on trait means was assessed while keeping cross as a fixed term in each model (including them in $\tau$ of Equation (1)). The linear influence of temperature was tested for by including the fixed covariate 'degree-days'. The influence of approximated differences in oxygen supply among families was tested for by including the fixed covariate 'density' (see above), which, however, was non-significant in all models and therefore removed.

Variation among dams (maternal environmental variance), among familial compartments (common familial environmental variance), and among tanks (common tank environmental variance) were accounted for by including identifications of 'dam', 'family' and 'tank' as random effects (in $u_{1}$ of Equation (1)). Further, additive genetic variance among individuals as predicted by the inverse additive relationship numerator matrix based on the complete four-generation pedigree) was accounted for by including identifications of 'animal' as random effects (in $u_{2}$ of Equation (1)). Such an animal model corrects for genetic relationships in unbalanced designs with relationship ties among individuals and increases the accuracy of fixed parameter mean estimates and their standard errors (Komender and Hoeschele, 1989).

Heterogeneous variances might be present among crosses due to segregation (Hayman, 1958; Piepho and Möhring, 2010) and they might be present between both parental populations. Hence, each random term was tested for variance heterogeneity among the four maternal genotype levels (terms 'dam', residuals), among the nine (non-reciprocal) offspring genotype levels (terms 'family', 'animal', residuals) or among the 14 reciprocal offspring crosses (residuals). However, heterogeneity was only observed for residuals (Supplementary Table S3). For other random terms, such a heterogeneous variance structure either did not improve the model fit or resulted in estimation problems for variances related to low DD dam sample sizes. As a consequence, variances for random terms other than residuals were estimated across all families.

For each of the six traits, 14 diploid outbreeding effects (based on Mather and Jinks, 1982; Supplementary Table S1) were estimated by including them as fixed continuous covariates in the trait models (in $\tau$ of Equation (1)). Effects fitted were the reference mean $(\hat{m})$, additive $(\hat{d})$, dominant $(\hat{h})$, additive-byadditive $(\hat{i})$, additive-by-dominant $(\hat{j})$, dominant-by-dominant $(\hat{l})$, maternal additive $\left(\hat{d}_{m}\right)$, maternal dominant $\left(\hat{h}_{m}\right)$, maternal additive-by-maternal additive $\left(\hat{i}_{m}\right)$, maternal dominant-by-maternal dominant $\left(\hat{l}_{m}\right)$, additiveby-maternal additive $\left(\hat{d} . d_{m}\right)$, dominant-by-maternal additive $\left(\hat{h} . d_{m}\right)$, dominant-by-maternal dominant $\left(\hat{h} . h_{m}\right)$ and additive-by-maternal dominant $\left(\hat{d} . h_{m}\right)$ effects.
The model fit when including each outbreeding effect was assessed by including a lack-of-fit term (the reciprocal cross term). Any model that was non-significant for the lack-of-fit term $(P>0.05$, Wald's F-tests) was regarded as fitting the data. This model selection strategy for cross mean analysis was suggested by Piepho and Möhring (2010). Outbreeding effects were first tested one-by-one and then by combinations of significant effects $(P<0.05$, Wald's F-tests). If this resulted in several models with equal numbers of significant effects fitting the cross means, we reported all of them.

Yolk conversion efficiency and yolk percentage. Gross yolk dry mass conversion efficiency between hatch and time of first feeding $\left(E_{y o l k}\right)$ was calculated for every family with:

$$
E_{\text {yolk }}=\left(B_{f}-B_{a}\right) /\left(Y_{a}-Y_{f}\right)
$$

in which average dry masses are: $B_{f}$ fry body; $B_{a}$, alevin body; $Y_{a}$, alevin yolk; and $Y_{f}$, fry yolk. $E_{\text {yolk }}$ (logit-transformed) was analysed using a LMM with cross as a fixed term, dam as a random term and a Gaussian residual distribution. Furthermore, the correlation between eyed egg size and $E_{\text {yolk }}$ was tested using Pearson's product moment correlation.

A decrease of yolk mass and an increase in body mass between hatch and first feeding stages might also vary among crosses due to differences in yolk depletion rates. To test this, the proportions of yolk to total dry mass (logittransformed) of all individuals were analysed by a LMM with the fixed terms of cross, stage, the interaction of cross-by-stage and the fixed continuous covariates of degree-days at stage and density at stage. Evaluated by sequential model fitting, the random term dam-by-stage was fitted with a diagonal variance structure, permitting different among-dam variances between stages. The random term family-by-stage was fitted with an unstructured (co)variance structure permitting a covariance between familial environmental effects between stages. Gaussian residuals were allowed to have independent strata among the 28 levels of cross-by-stage.

For all models, significance $(P<0.05)$ of fixed terms (including outbreeding effects) was tested using conditional Wald-type F-tests with denominator degrees of freedom approximated following Kenward and Roger (1997). Significances of random terms and (co)variance structures were approximated by using Residual Maximum Likelihood log-likelihood ratio tests between nested models with $P<0.1$ as the critical alpha level. Only significant effects and (co)variance structures were contained in the models except for the family term (the basic experimental unit) and the animal term (accounting for correlated individual effects), which were always contained, if applicable. Means and their s.es. were jointly obtained as marginal model predictions. Contrasts between marginal means were conducted by Student's $t$-tests with degrees of freedom approximated as for F-tests and $P$-values were Bonferroniadjusted. All analyses were conducted using Residual Maximum Likelihood routines in ASReml-R 3.0 (Butler et al., 2009).

\section{RESULTS}

Twenty-five of all 64 dams had non-viable or poor-quality eggs (mostly DD dams) of which no or few individuals could be sampled. A necessary spawning synchronisation might have resulted in a nonoptimal spawning timing, especially for DD dams, or our laboratory conditions had population-specific effects on fertility. To exclude such potential effects from our results, only data from dams with sufficient sample size ( $>3$ per family) were used in the analyses.

\section{Maternal traits}

Among the four dam crosses, average fork lengths of fertile dams were different $\left(\mathrm{F}_{3 / 35}=7.4, P<0.001\right)$ but differences for body masses were non-significant $\left(\mathrm{F}_{3 / 35}=2.8, P=0.056\right)$; WW dams were shorter (three

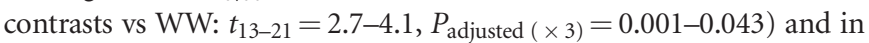
their tendency lighter than other dams (Figures $3 \mathrm{a}$ and $\mathrm{b}$ ). Similar average sizes were obtained with 2-8 times larger sample sizes per dam cross when using data from all mature females available at spawning (Supplementary Table S2), indicating that size data on dams were representative. 

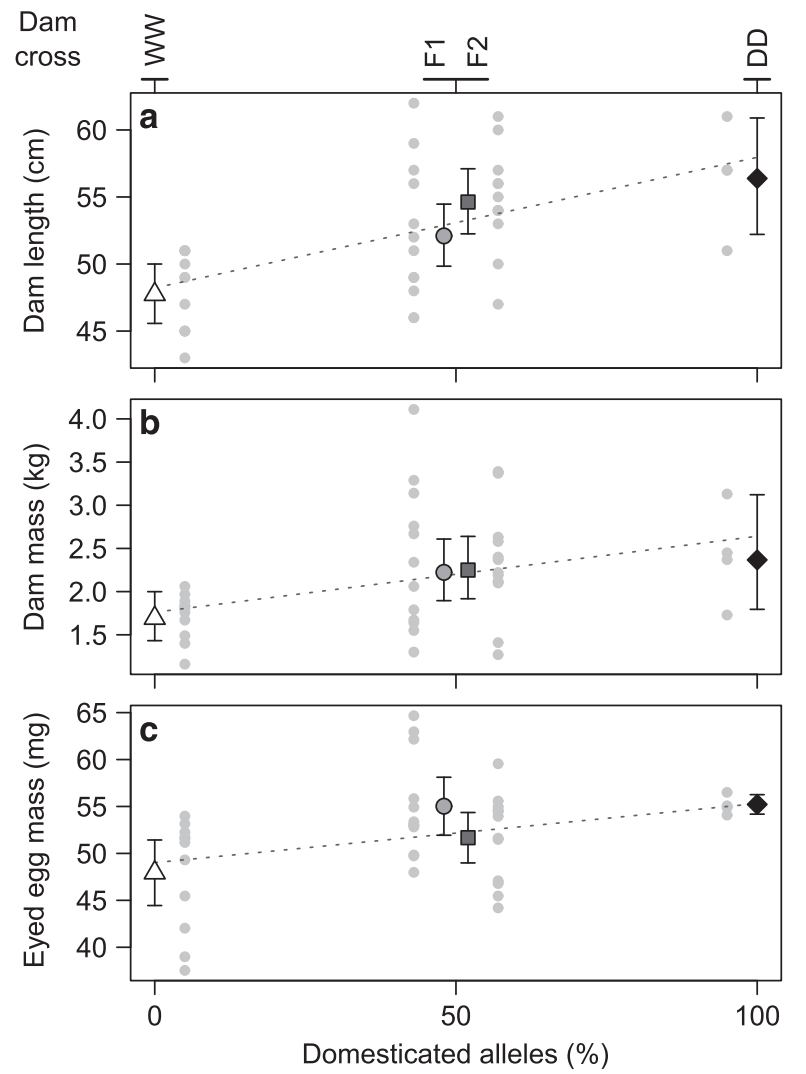

Figure 3 Average re-transformed fork length (a), re-transformed body mass (b) and average eyed egg dry mass (c) from the four 2005 generation dam crosses of wild (WW), white triangles; domesticated (DD), black diamonds; first-generation hybrid (F1), light grey circles; and second-generation hybrid (F2), dark grey squares, Atlantic salmon used to create the 2009 generation. Error bars depict approximate 95\% confidence intervals. For each trait, the dam crosses have been arranged by percentage of domesticated alleles. The vertically arranged grey dots beside each cross mean depict individual data (a, b) or dam mean across families (c). The dotted line in each panel represents the reference mean under a complete additive genetic architecture. Symbols for hybrids (at 50\% domesticated alleles) have been slightly off-set to improve depiction.

Average eyed egg dry mass was different among dam crosses $\left(\mathrm{F}_{3 / 17.9}=6.1, \quad P=0.005\right)$ inferred by a model that accounted for heterogeneous among-dam variances for the four dam crosses and for overall among-family variance (65-69\% (6\% for DD) and 1\% ( $4 \%$ for DD) of the total variance per dam cross, respectively). Domesticated and F1 hybrid dams had similar average egg sizes that were both larger than WW dam eggs (DD vs WW: $t_{11.5}=3.9$, $P_{\text {adjusted }(\times 6)}=0.014$; F1 vs WW: $\left.t_{20.4}=3.0, P_{\text {adjusted }(\times 6)}=0.044\right)$; all other cross mean comparisons were non-significant $\left(t_{13-21.6}=1.7-2.4\right.$, $\left.P_{\text {adjusted }(\times 6)}=0.187-1\right)$. WW dams had the smallest eggs and F2 hybrid dam eggs were fitting the midparental value (Figure $3 \mathrm{c}$ ). Averaged egg size correlated positively with dam length and dam mass ( $\left.r_{\text {length }}=0.40, P=0.012 ; r_{\text {mass }}=0.49, P=0.001\right)$.

\section{Survival and incubation period}

Offspring survival between fertilisation and the fry stage did not differ among offspring crosses $\left(\mathrm{F}_{13 / 12.8}=1.2, P=0.388\right)$, but means varied between $12 \%$ and $54 \%$ with tendencies of lower survival for DD dam families and higher survival for families from both hybrid dam crosses
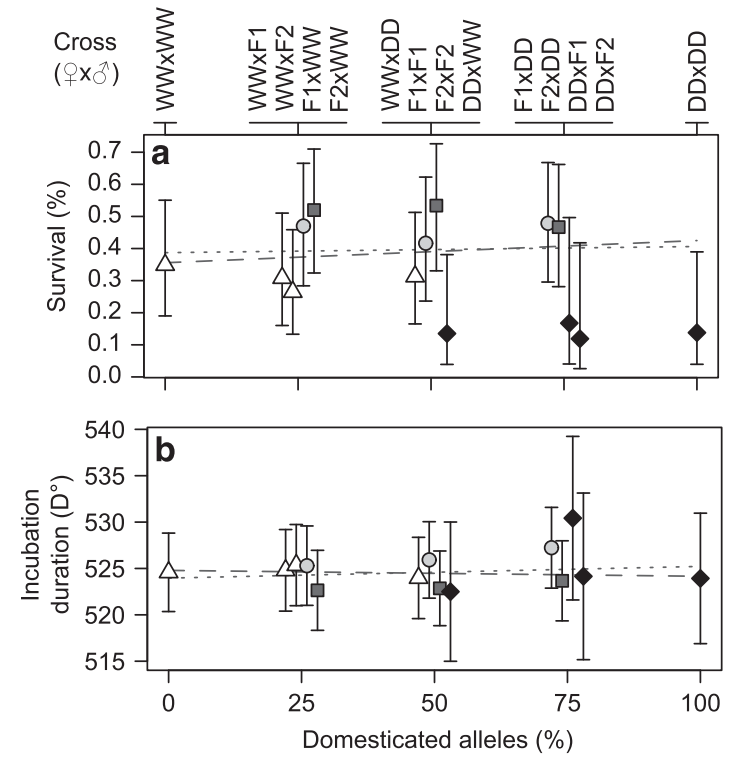

Figure 4 Average re-transformed percentage of surviving individuals between fertilisation and time of first feeding (a), and average incubation duration until 50\% hatch in cumulative degree-days (b) for 14 reciprocal crosses of the 2009 generation between wild and domesticated Atlantic salmon. Error bars depict approximate 95\% confidence intervals. The two lines in each panel represent the reference mean under either an additive (dotted line) or a maternal additive (dashed line) genetic model. Symbols for hybrids and backcrosses (at 25, 50 and 75\% domesticated alleles) have been slightly off-set to improve depiction. Symbols and colours indicate maternal cross with wild (WW), white triangles; domesticated (DD), black diamonds; first-generation hybrid (F1), light grey circles; and secondgeneration hybrid (F2), dark grey squares. Reciprocal crosses are indicated above the panels as dam cross by sire cross.

(Figure 4a). Among-dam variance accounted for $76-100 \%$ of the total variance in survival per cross, indicating little influence of offspring genotype.

The incubation period until 50\% hatch of families lasted between 498.3 and $540.2 \mathrm{D}^{\circ}$ and was not associated with familial density, egg mass or biomass $\left(P_{\text {density }}=0.369, P_{\text {egg mass }}=0.303, P_{\text {biomass }}=0.982\right)$, which suggests sufficient oxygenation and no influence of egg size on incubation period. However, among-tank variance was significant $\left(\chi^{2}{ }_{1}=14.3, P<0.001\right)$, indicating systematic environmental influence on degree-days until hatch and emphasising the importance of the conducted spatial randomisation of units. Differences among offspring crosses were minor (Figure $4 \mathrm{~b}$ ) and non-significant $\left(F_{13 / 128.3}=0.7, P=0.771\right)$. In a LMM with a fitted common intercept among all families (mean \pm s.e.: $524.5 \pm 1.1 \mathrm{D}^{\circ}$ ), among-dam and among-tank variation accounted for $63 \%$ and $14 \%$, respectively, of the total variance in the incubation period.

\section{Cross-means analyses of alevin and fry traits}

Among-dam variance was the most important variance component for the six traits of length, body dry mass and yolk dry mass of alevins and fry, comprising between 22-34\% (alevin length) and 54-93\% (alevin yolk mass) of the total variance per cross. Among-family variance was significant for most traits, except for alevin yolk mass, comprising between 1\% (alevin yolk mass) and 9-13\% (alevin length) of the total variance per cross. 
Conversely, shared among-tank variance made up a small percentage of the total variance and was only significant for alevin body mass, comprising $3-6 \%$ of the total variance per cross. The additive genetic variance estimated for 'animal' varied among traits from zero (alevin yolk and fry body mass) to $10-20 \%$ of the total variance per cross (alevin body mass). See Supplementary Tables S3a-f for further model details.

Alevin standard length at hatch was positively associated with degree-days (effect \pm s.e.: $\quad 0.021 \pm 0.006 \mathrm{~mm} \mathrm{D}^{\circ-1}, \quad \mathrm{~F}_{1 / 155.6}=11.7$, $P=0.001)$. The common degree-day-adjusted cross means were different $\left(\mathrm{F}_{13 / 101.5}=3.4, P<0.001\right)$ where the $\mathrm{DD} \times \mathrm{F} 1$ cross was longer than most others (Figure $5 \mathrm{a}$ ). No outbreeding effect was different from zero, with the maternal additive effect having the lowest probability $(P=0.081)$, and no fit to the cross means could be obtained (Table 2; Supplementary Table S4a). Omitting the DD $\times$ F1 cross strongly reduced the statistical support for among-cross differences $\left(\mathrm{F}_{12 / 92.3}=1.9, P=0.050\right)$ and increased it for degree-days $\left(\mathrm{F}_{1 / 150.4}=13.2, P<0.001\right)$.

Fry standard length at the time of first feeding was not associated with any tested continuous covariate. Differences among crosses were sufficiently explained by either the maternal additive, the additive or the additive-by-dominant effect (Table 2; Supplementary Table S4b). However, cross mean differences were non-significant $\left(\mathrm{F}_{13 / 99.6}=1.8\right.$, $P=0.058)$ but pooled by dam cross differences among means were significant $\left(\mathrm{F}_{3 / 38.2}=3.1, P=0.042\right)$, which might support the maternal additive effect most. Increasing percentage of domesticated alleles of dams resulted in increasing length of their offspring (Figure 5b).

Alevin body dry mass was positively influenced by degree-days (effect \pm s.e.: $0.026 \pm 0.0053 \mathrm{mg} \mathrm{D}^{\circ-1}, \mathrm{~F}_{1 / 154.2}=23.6, P<0.001$ ). Only the maternal additive outbreeding effect with a small effect size was significant (Table 2) but obtained under a significant lack-of-fit to the cross means ( $P_{\text {lack-of-fit }}=0.003$, Supplementary Table S4c). The
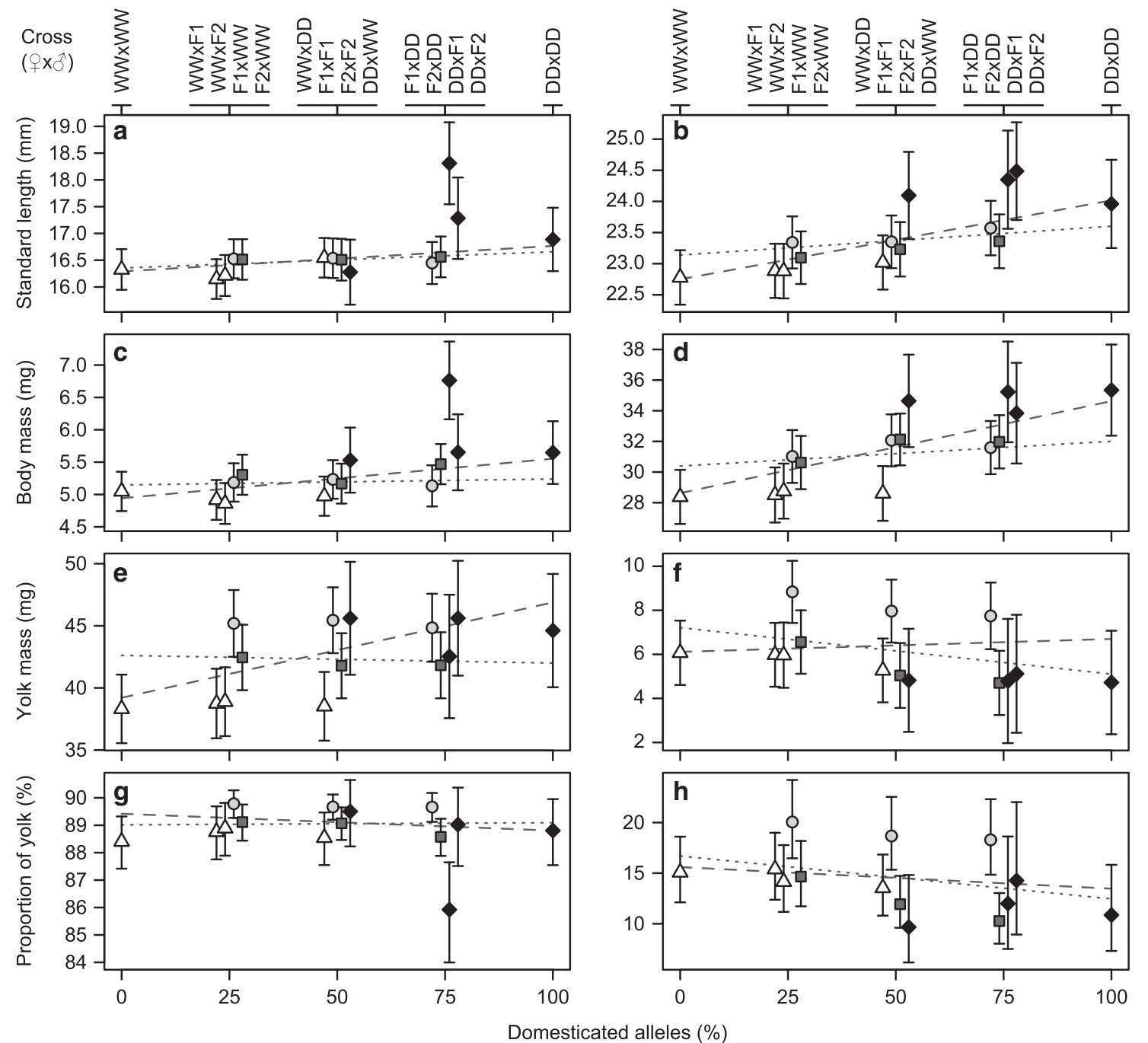

Figure 5 Estimated trait means with approximate 95\% confidence intervals (error bars) for 14 reciprocal crosses of the 2009 generation between wild and domesticated Atlantic salmon for alevins at hatch (left panels, a, c, e, g) and fry at time of first feeding (right panels, b, d, f, h). Displayed traits are standard length (a, b), body dry mass (c, d), yolk dry mass (e, f) and proportion of yolk to total dry mass ( $\mathbf{g}$, h). The two lines in each panel represent the reference mean under either an additive (dotted line) or a maternal additive (dashed line) genetic model. Symbols for hybrids and backcrosses (at 25 , 50 and $75 \%$ domesticated alleles) have been slightly off-set to improve depiction. Symbols and colours indicate maternal cross with wild (WW), white triangles; domesticated (DD), black diamonds; first-generation hybrid (F1), light grey circles; and second-generation hybrid (F2), dark grey squares. Reciprocal crosses are indicated above the panels as dam by sire cross. 
Table 2 Trait mean $(\hat{m})$ and outbreeding effects both with s.e., F-values (with degrees of freedom/denominator degrees of freedom) and $P$-values for the best models fitted to 14 cross means for each of the six traits for Atlantic salmon alevins at hatch and fry at the time of first feeding

\begin{tabular}{|c|c|c|c|c|}
\hline Traita & $\hat{m}($ s.e. $)$ & Effect(s) (s.e.) & $\mathrm{F}(d f / d d f)$ & $\mathrm{P}$ \\
\hline levin length ${ }^{b}$ & $16.4(0.1)$ & - & - & - \\
\hline ry length I & $23.3(0.12)$ & $\hat{d}=-0.24(0$ & $7.7(1 / 11.6)$ & 0.017 \\
\hline ry length II & $23.3(0.13)$ & $\hat{j}=-3.3(1.6)$ & $4.3(1 / 130)$ & 0.041 \\
\hline Fry length III & $23.3(0.12)$ & $\hat{d}_{m}=-0.5$ & $8.2(1 / 3$ & 0.007 \\
\hline Alevin body mass ${ }^{c}$ & $5.3(0.08)$ & $\hat{d}_{m}=-$ & $7.5(1 / 38.8)$ & 0.00 \\
\hline \multirow[t]{2}{*}{ Fry body mass } & $31.7(0.49)$ & $\hat{d}=-0.7(0$ & $6.6(1 / 9.5)$ & 0.029 \\
\hline & & $\hat{d}_{m}=-2$ & 12.9 & 0.001 \\
\hline levin yolk mass I & 43.0 & $\hat{d}_{m}=-3.8(1.3)$ & $9.1(1 / 36.7)$ & 0.005 \\
\hline levin yolk mass II & $40.1(1.2)$ & $\hat{h}_{m}=4.9(1.8)$ & $6.6(1 / 37.2)$ & 0.014 \\
\hline Alevin yolk mass III & $40.5(1.0)$ & $\hat{I}_{m}=4.8(1$ & $6.8(1 / 37.4)$ & 0.013 \\
\hline Alevin yolk mass IV & $41.2(0.91)$ & $\hat{h} . h_{m}=5.4(2.2)$ & $6.2(1 / 129.3)$ & 0.014 \\
\hline Fry yolk mass I & $6.3(0.45)$ & $\hat{j}=1.9(0.60)$ & $10.5(1 / 150.8)$ & 0.001 \\
\hline Fry yo & 5.3 & $\hat{I}_{m}=2.9$ & $12.6(1 / 42.4)$ & $<0.001$ \\
\hline Fry yolk mass III & $5.2(0.54)$ & $\hat{h} . h_{m}=5.6(1.5)$ & $13.9(1 / 68.9)$ & $<0.00$ \\
\hline
\end{tabular}

Abbreviations of outbreeding effects are used as in the text.

a Units are $\mathrm{mm}$ for length and $\mathrm{mg}$ for mass. If more than one model fit cross means, the different models for a trait are indicated by roman numerals.

${ }^{b}$ For alevin length, no effect was significantly different from zero and no fit to the cross means could be obtained.

'For alevin body mass, no significant fit to the cross means could be obtained and results are given for the model with effects significantly different from zero best fitting the cross means.

degree-day-adjusted cross-means pattern mirrored the inference of a maternal additive effect with the effect of increasing percentage of domesticated alleles of dams, resulting in increasing body mass of their offspring (Figure 5c). However, although differences among offspring cross means were significant $\left(F_{13 / 84.9}=3.2, P=0.001\right)$, no indications of offspring genotype effects appeared to be present except that the DD $\times$ F1 cross was divergent from most others (Figure $5 \mathrm{c}$ ). Omitting the $\mathrm{DD} \times \mathrm{F} 1$ cross resulted in a loss of significance for among-cross differences $\left(\mathrm{F}_{12 / 85.5}=1.6, P=0.118\right)$ but not for degreedays $\left(\mathrm{F}_{1 / 153.4}=25, P<0.001\right)$.

Fry body dry mass was not predicted by any continuous covariate. The cross means were different $\left(\mathrm{F}_{13 / 145.1}=3.5, P<0.001\right)$ and best predicted by the combination of the additive with the maternal additive effect (Table 2, Supplementary Table 4d). The cross-means pattern largely followed the pattern of length at hatch and confirmed the inferred outbreeding effects (Figure 5d). However, the additive effect, that is, decreasing mass with decreasing percentage of DD alleles, was small and unidirectional, exhibited in backcrosses with WW sires only.

Alevin yolk dry mass was not predicted by any continuous covariate. None of the offspring outbreeding effects were significant, but all maternal outbreeding effects and one epistatic maternaloffspring effect were significant (Supplementary Table 4e). Models that fit the significantly different cross means $\left(\mathrm{F}_{13 / 86.2}=2.2\right.$, $P=0.014$ ) included either the maternal additive, the maternal dominant, maternal dominant-by-dominant or the epistatic offspring dominant-by-maternal dominant effect (Table 2, Supplementary Table 4e). Yolk mass among crosses largely mirrored the pattern of egg size by dam cross with the largest yolks possessed by families from $\mathrm{DD}$ and $\mathrm{F} 1$ hybrid dams (Figure $5 \mathrm{e}$ ). However, the $\mathrm{DD} \times \mathrm{F} 1$ backcross exhibited a somewhat lower yolk dry mass relative to other DD dam crosses.
Fry yolk dry mass was negatively influenced by degree-days (effect \pm s.e.: $-0.07 \pm 0.016 \mathrm{mg} \mathrm{D}^{\circ-1}, \mathrm{~F}_{1 / 128.8}=21.8, P<0.001$ ). The pattern of degree-day-adjusted, different cross means $\left(\mathrm{F}_{13 / 108.8}=2.5\right.$, $P=0.005)$ was best predicted by either the maternal dominant-bydominant, the offspring additive-by-dominant or the epistatic offspring dominant-by-maternal dominant effect (Table 2, Supplementary Table 4f). These effects were mirrored by the crossmeans pattern, although the additive or additive-by-dominant effect (that is, modifying backcross means relative to maternal cross effects) was more pronounced where involving wild parents (Figure 5f). Also, the dominant-by-maternal dominant pattern (that is, changing all F1 and F2 hybrid dam offspring means from the midparental value, but F2 hybrid dam offspring only one fourth as much) was not recognisable for crosses from F2 hybrid dams (Figure 5f).

\section{Yolk conversion efficiency and yolk percentage}

Familial gross conversion efficiency did not differ among the 14 crosses $\left(\mathrm{F}_{13 / 169}=0.6, P=0.820\right.$, overall $E_{\text {yolk }} \pm$ s.e.: $\left.0.731 \pm 0.005\right)$ and among-dam variance was non-significant $\left(\chi^{2}{ }_{1}=0, P=1\right)$. Conversion efficiency did not correlate with egg size $(r=-0.021, P=0.773)$.

The logit-transformed proportion of yolk to total dry mass (percentage of yolk) was negatively influenced by degree-days at sampling for both stages (alevin: $\mathrm{F}_{1 / 159.8}=26.5, P<0.001$; fry: $\mathrm{F}_{1 / 131}=35.2, P<0.001$ ) but not by density (alevin: $\mathrm{F}_{1 / 163.2}=0.001$, $P=0.944$; fry: $\mathrm{F}_{1 / 172.9}=1.4, P=0.236$ ). Re-transformed decrease in the proportion of yolk mass with increasing degree-days was more than three times faster for fry than for alevins (effects \pm s.e.: $-0.062 \pm 0.030 \% \mathrm{D}^{\circ-1}$ in alevin vs $-0.221 \pm 0.078 \% \mathrm{D}^{\circ-1}$ in fry). Stage-specific degree-day-adjusted percentage of yolk differed among crosses $\left(\mathrm{F}_{13 / 128.7}=4.2, P<0.001\right)$, between stages $\left(\mathrm{F}_{1 / 38.4}=3303\right.$, $P<0.001)$ and for the interaction of cross-by-stage $\left(F_{13 / 140.8}=2.9\right.$, $P=0.001)$. A weak indication for a correlation between both stages was detected for family random effects $\left(\chi^{2}{ }_{1}=3.0, \quad P=0.084\right.$, $\rho=0.31$ ), but not for dam random effects, indicating a potential for the presence of persistent common familial environmental influence on yolk proportion.

Between the alevin and the fry stage and when averaged by dam cross, offspring from F1 hybrid dams had reduced their yolk proportion by $71 \%$, those from WW dams by $74 \%$ and those from both F2 and DD dams by $77 \%$, indicating different yolk-depletion rates. At hatch, most crosses had an equal average amount of around $89 \%$ yolk (Figure $5 \mathrm{~g}$ ). For fry, the pattern was more diverse and percentage of yolk among crosses differed twofold (9-20\%; Figure $5 \mathrm{~h}$ ). Most crosses showed decreasing percentages of yolk mass with increasing percentages of domesticated alleles. However, all three crosses involving F1 hybrid dams had the highest percentages of residual yolk mass (18-20\%), on average 51\% larger than those of other crosses and $31 \%$ higher than predicted under an additive model $(14.5 \%)$

\section{DISCUSSION}

We did not detect any differences in survival, hatch timing and yolk conversion efficiency between two divergent Atlantic salmon populations. Further, even after two rounds of recombination, no effects of outbreeding on means were detected for these traits. This might suggest equality or at least compatibility of trait-specific alleles as expressed under our laboratory conditions. Nevertheless, results on other traits revealed outbreeding effects that are likely to influence offspring fitness in early life. Strong individual maternal effects were detected for most offspring traits in accordance with previous studies. Although accounting for this individual among-dam variation, we 
detected maternal outbreeding effects on means, that is, the differences in population-specific maternal effects and their change in hybrid dams that presumably underlie the genetic architecture of divergent alleles from both populations. Furthermore, we detected additive and non-additive outbreeding effects at the offspring genotype level and an indication of epistasis between the maternal and the offspring level. Altogether, these results render the predictability of phenotypes and their potential fitness resulting from outbreeding difficult. As we controlled for parental and offspring environments and ages, we concluded that all inferred outbreeding effects are genetically based.

\section{Maternal traits}

The presence of positive correlations between female size and egg size, and between egg size and early offspring size, has been well documented in salmonids (Beacham et al., 1985; Ojanguren et al., 1996). Between the wild and domesticated populations used in the present study, large differences in maternal body size existed (see also Fraser et al., 2010). In our four dam crosses, egg size also generally increased with maternal body size. Nonetheless, egg size appeared to be controlled by additional mechanisms than just body size. F1 hybrid dams were shorter than F2 hybrid dams. However, shorter F1 hybrid dams produced larger eggs than longer F2 hybrid dams, albeit none of the differences between F1 and F2 hybrid dams were significant. The larger F1 than F2 dam egg size suggests the presence of a non-additive genetic component for this trait, independent of maternal body size, which yet remains to be evaluated.

\section{Maternal outbreeding effects}

For four of six offspring traits analysed, the maternal additive outbreeding effect underlay, alone or in tandem with other effects, the cross means. This suggests that the maternal genetic additive architecture for these traits is at least partly de-coupled from the detected (dominant-by-) maternal-dominant outbreeding effect for yolk mass. Yet, the maternal additive and maternal dominant outbreeding effects almost certainly had consequences for offspring traits as a result of their combination. Offsprings from both F1 and F2 hybrid dams had nearly intermediate (midparental) body length and mass at both hatch and the time of first feeding, but originated either from large eggs spawned by F1 hybrid dams or from intermediate-size eggs spawned by F2 hybrid dams. For offspring only from F1 hybrid dams, a presumed mismatch of large egg size and intermediate offspring size resulted in a relatively larger yolk mass. This larger yolk mass at both hatch and at the time of first feeding was then exhibited as maternal-dominant outbreeding effect.

Two different types of mismatches might explain the large residual yolk mass evident in F1 hybrid dam offspring, although the causal physiological mechanisms are unknown. Firstly, F1 hybrid dam offspring developed slower than other offspring, which would be supported by their slower yolk proportion depletion rate. Secondly, F1 hybrid dam offspring developed equally fast as other offspring but had larger residual yolks. In the first case, F1 hybrid dam offspring might reach a body size that is proportional to their egg size later in time (i.e., they might continue to grow longer solely on yolk reserves than others) but also emerge later from the gravel than other crosses. In the second case, F1 hybrid dam offspring will have a body size that is smaller than predicted by their egg size and might emerge with large residual yolk at the same time as other offspring.

Unfortunately, the literature is equivocal on what represents salmon development in terms of residual yolk. Some authors use the percentage of body mass to total mass as an index of development to predict first feeding at $97 \%$ body wet mass (Thorpe et al., 1984). Yet, fry in the wild might start feeding in the gravel (GustafsonMarjanen and Dowse, 1983) and early emerging fry have externally visible yolks (Garcia de Leaniz et al., 2000). In our study, externally visible yolks were observed on some individuals upon dissection of fry. Nevertheless, before fry sampling we observed that all offspring exhibited swim-up behaviour, which is usually associated with first feeding. Lastly, degree-days for fry had no effect on body mass but a negative effect on yolk mass, making it possible that a pre-determined size had been attained and only movement caused further yolkdepletion. Hence, it is likely that all fry were fully developed. Regardless what is seen as an approximation of development, judged on residual yolk percentage, development was slower or, more likely, imbalanced between egg/yolk mass and body mass in F1 hybrid dam offspring relative to other crosses.

There are possible consequences resulting from maternal outbreeding effects for fitness-related relationships between female size and egg size, egg number and offspring size - a central concept in life history evolution (Roff, 1992; Rollinson and Hutchings, 2013). The phase following fry emergence has been identified as a 'critical period' because of high mortalities caused by predation and starvation (Elliott, 1990; Einum and Fleming, 2000). It is generally accepted that larger fry are fitter through advantages in feeding territory acquisition (Elliott, 1990; Cutts et al., 1999), with larger sizes of only a few per cent resulting in dominance advantages (Berejikian et al., 1996), and predator avoidance (Mogensen and Hutchings, 2012). Large eggs result in large offspring but are under evolutionary constraints because of the trade-off between egg size and egg number (Sargent et al., 1987). Time of emergence may also affect fitness with earlier emerging fry advantageously finding territories first (Cutts et al., 1999; Mogensen and Hutchings, 2012) but being at a disadvantage under predation (Brännäs, 1995).

In fishes, it is generally assumed that egg size is adaptive (Kamler, 1992) with egg number adjusted for environmentally influenced available maternal energy (Scott, 1962). Hence, maternal outbreeding effects detected in the present study have the potential to either result in smaller wild-domesticated offspring size per egg size or delayed emergence when hybrid dams are involved. The latter might compromise fitness more severely (Einum and Fleming, 2000) and both might shift trait values in adapted populations and lead to outbreeding depression. Underdeveloped fry may also be more readily preyed upon because of reduced swimming ability (Bams, 1967), but they may survive longer under food limitation by virtue of their larger residual yolk. Conversely, all domesticated dam offspring appeared to have a faster yolk-depletion rate. Accordingly, predation or starvationrelated fitness effects, even those resulting from simple maternal additive outbreeding effects, are likely to be environment-specific and these effects will additionally differ through maternal non-additive outbreeding effects on hybrid generation-specific phenotypes.

\section{Offspring outbreeding effects and epistasis}

We detected offspring outbreeding effects and indications of epistatic interactions between offspring and maternal outbreeding effects. Hence, offspring traits and development are governed by the offspring genotype and possibly by interactions between maternal and offspring genotypes. These effects likely act in addition to the major maternal effects that are a correlate of maternal genotype, egg size, and eggdeposited factors. Outbreeding effects at the offspring level were, apart from a presumed epistatic interaction with maternal effects, absent at the alevin stage but present for all traits at the fry stage. This growing 
influence of offspring genotype towards first feeding on means is in accordance with other studies on variances (Heath et al., 1999; Perry et al., 2004; Aykanat et al., 2012).

\section{Remarks on study-related limitations}

In creating the 2005 generation, we had to obtain additional gametes from the WW source population and this might have caused a genetic heterogeneity. Yet, we did not observe a closer similarity between 2005 F1 and WW dams (contribution of new breeders in both) than between 2005 F2 and WW dams (no contribution of new breeders in F2). Furthermore, crosses generated for this study in 2009 were derived entirely from parents that had shared a lifetime common laboratory environment, which likely removed previous heterogeneous environmental effects.

Low survival of offspring from domesticated dams resulted in the availability of only few DD families. Of the four DD dams from which we collected familial offspring data, two were full-sibs and two others were maternal half-sibs. This may have introduced a bias in all DD dam offspring data by, for example, their very small variation in egg size relative to other dam crosses. We cannot determine whether the small variation is a result of the domestication process (as suggested for variation in growth; Solberg et al., 2013) or of the close kinship among dams. However, it was not possible to account in our analyses for dam cross-specific variances (except for egg size) because of nonestimable DD dam cross variances. Hence, we had to jointly estimate among-dam variances across all families. As a result, fitted variances for DD dam offspring were likely larger than in reality and this might have resulted in conservative inferences.

We concluded that either maternal or epistatic offspring-bymaternal effects underlie alevin yolk mass and that fry yolk mass is determined by either epistatic offspring, epistatic maternal or by epistatic offspring-by-maternal effects. Although from graphical examination it appears necessary to explain cross means by combinations of effects at the maternal and offspring level, respective crossmeans patterns did not agree with means predicted by each single effect. This lack of concordance might have been caused by (i) too large variation for cross means to detect possible effect combinations (also because of few DD dam cross families), (ii) our use of a simple diallelic model with epistasis between two loci per level, whereas quantitative traits probably underlie several loci, each with many alleles. Moreover, (iii) Atlantic salmon possess many gene duplications due to a presumed family-specific genome duplication event, which might render a diploid model partly inappropriate (Fraser et al., 2010).

\section{Conclusion and implications}

We found no indications of a typical break-up of epistatic coadapted offspring genes between two divergent salmon populations. However, our work revealed a complex set of additive and non-additive effects at both the offspring and the maternal genotype level and potential epistasis between both. As a consequence, phenotypes resulting from outbreeding between the wild and domesticated salmon are not readily predictable, but vary with the number of hybrid generations of both mothers and offspring, and particular environmental conditions will govern their fitness.

Our results nevertheless suggest that non-additive maternal outbreeding effects (known as maternal heterosis in animal breeding), and potentially their interaction with additive maternal and direct genetic effects, might have important roles in outbreeding-related phenomena of wild populations, such as generation-specific heterosis and outbreeding depression. The interplay of maternal additive (for offspring body size) and maternal dominant effects (for yolk size) in F1 hybrid dam offspring suggested here might be regarded as empirical evidence of the mechanistic breakdown of maternally controlled coadapted offspring traits. Such a breakdown might affect many species in which direct or indirect genetic-based maternal effects occur and its consideration is warranted in evolutionary, conservation or economical-agricultural oriented studies. These investigations should be conducted using reciprocal breeding designs and hybrid parents to allow for the distinction between the maternal and offspring level as the origin of possible outbreeding effects.

\section{DATA ARCHIVING}

Data deposited in the Dryad repository: doi:10.5061/dryad.9cs2v.

\section{CONFLICT OF INTEREST}

The authors declare no conflict of interest.

\section{ACKNOWLEDGEMENTS}

This work was approved by the Dalhousie University Committee on Laboratory Animals and followed guidelines of the Canadian Council on Animal Care (CCAC, 2005). We thank Robin Waples and an anonymous reviewer for improving this manuscript. We also thank DFO staff from Coldbrook and Mersey facilities, especially Beth Lenentine and John Whitelaw, and the Aquatron facility staff. For invaluable experimental help, we thank Matthew Yates, Katharina Bremer, Samantha Hamilton, Njal Rollinson, Aimee and Corey Houde, Dave Keith and Stephanie Mogensen. The research was supported by NSERC Strategic and Discovery Grants to JAH and by an NSERC Postdoctoral Fellowship to DJF.

Aykanat T, Bryden CA, Heath DD (2012). Sex-biased genetic component distribution among populations: additive genetic and maternal contributions to phenotypic differences among populations of Chinook salmon. J Evol Biol 25: 682-690.

Bams RA (1967). Differences in performance of naturally and artificially propagated sockeye salmon migrant fry as measured with swimming and predation tests. J Fish Res Board Can 24: 1117-1153.

Beacham TD, Withler FC, Morley RB (1985). Effect of egg size on incubation time and alevin and fry size in chum salmon (Oncorhynchus keta) and coho salmon (Oncorhynchus kisutch). Can J Zool 63: 847-850.

Berejikian BA, Mathews SB, Quinn TP (1996). Effects of hatchery and wild ancestry and rearing environments on the development of agonistic behavior in steelhead trout (Oncorhynchus mykiss) fry. Can J Fish Aquat Sci 53: 2004-2014.

Brännäs E (1995). First access to territorial space and exposure to strong predation pressure: a conflict in early emerging Atlantic salmon (Salmo salar L.) fry. Evol Ecol 9 411-420.

Burke JM, Arnold ML (2001). Genetics and the fitness of hybrids. Annu Rev Genet 35 31-52.

Butler DG, Cullis BR, Gilmour AR, Gogel BJ (2009). Mixed Models for S Language Environments ASReml-R Reference Manual. Queensland Department of Primary Industries and Fisheries, NSW Department of Primary Industries: Brisbane, Australia.

CCAC (2005). CCAC Guidelines on the Care and Use of Fish in Research, Teaching and Testing. Canadian Council on Animal Care: Ottawa, Canada.

Cutts CJ, Metcalfe NB, Taylor AC (1999). Competitive asymmetries in territorial juvenile Atlantic salmon, Salmo salar. Oikos 86: 479-486.

Einum S, Fleming IA (2000). Selection against late emergence and small offspring in Atlantic salmon (Salmo salar). Evolution 54: 628-639.

Elliott JM (1990). Mechanisms responsible for population regulation in young migratory trout, Salmo trutta. III. The role of territorial behaviour. J Anim Ecol 59: 803-818.

Fraser DJ, Houde AL, Debes PV, O'Reilly P, Eddington JD, Hutchings JA (2010). Consequences of farmed-wild hybridization across divergent wild populations and multiple traits in salmon. Ecol App/ 20: 935-953.

Fraser DJ, Weir LK, Bernatchez L, Hansen MM, Taylor EB (2011). Extent and scale of local adaptation in salmonid fishes: review and meta-analysis. Heredity 106: 404-420.

Garcia de Leaniz C, Fraser N, Huntingford FA (2000). Variability in performance in wild Atlantic salmon, Salmo salar L., fry from a single redd. Fish Manage Ecol 7: 489-502.

Glebe BD (1998). Canadian Stock Assessment Secretariat, Research Document 98/ 157Fisheries and Oceans Canada: Ottawa, Canada, pp 9.

Green BS (2008). Maternal effects in fish populations. In: Sims DW (eds). Advances in Marine Biology, 1st edn. Elsevier Ltd.: UK. vol. 54, pp 1-105.

Gustafson-Marjanen KI, Dowse HB (1983). Seasonal and diel patterns of emergence from the redd of Atlantic salmon (Salmo salar) fry. Can J Fish Aquat Sci 40: 813-817. 
Hayman BI (1958). The separation of epistatic from additive and dominance variation in generation means. Heredity 12: 371-390.

Heath DD, Fox CW, Heath JW (1999). Maternal effects on offspring size: variation through early development of chinook salmon. Evolution 53: 1605-1611.

Houde ALS, Fraser DJ, O'Reilly P, Hutchings JA (2011). Maternal and paternal effects on fitness correlates in outbred and inbred Atlantic salmon (Salmo salar). Can J Fish Aquat Sci 68: 534-549.

Kamler E (1992). Early Life History of Fish: An Energetics Approach, 1st edn. Chapman \& Hall: London, UK.

Kane TR (1988). Relationship of temperature and time of initial feeding of Atlantic salmon. Prog Fish-Cult 50: 93-97.

Kenward MG, Roger JH (1997). Small sample inference for fixed effects from restricted maximum likelihood. Biometrics 53: 983-997.

Komender P, Hoeschele I (1989). Use of mixed-model methodology to improve estimation of crossbreeding parameters. Livest Prod Sci 21: 101-113.

Kruuk LE, Hadfield JD (2007). How to separate genetic and environmental causes of similarity between relatives. J Evol Biol 20: 1890-1903.

Lawlor JL, Dacanay A, Hutchings JA, Brown LL, Sperker SA (2008). Differences in pathogen resistance within and among cultured, conservation-dependent, and endangered populations of Atlantic salmon, Salmo salar L. Environ Biol Fishes 84: 69-78.

Lynch M (1991). The genetic interpretation of inbreeding depression and outbreeding depression. Evolution 45: 622-629.

Marr DHA (1963). The influence of surface contour on the behaviour of trout alevins $S$. trutta L. Anim Behav 11: 412.

Mather K, Jinks JL (1982). Biometrical Genetics: The Study of Continuous Variation3rd edn. Chapman and Hall: London, UK.

Mogensen S, Hutchings (2012). Maternal fitness consequences of interactions among agents of mortality in early life of salmonids. Can J Fish Aquat Sci 69: 1539-1555.

Mousseau TA, Fox CW (1998). The adaptive significance of maternal effects. Trends Ecol Evol 13: 403-407.
Ojanguren AF, Reyes-Gavilán FG, Braña F (1996). Effects of egg size on offspring development and fitness in brown trout, Salmo trutta L. Aquaculture 147: 9-20.

Perry GML, Audet C, Laplatte B, Bernatchez L (2004). Shifting patterns in genetic control at the embryo-alevin boundary in brook charr. Evolution 58: 2002-2012.

Piepho HP, Möhring J (2010). Generation means analysis using mixed models. Crop Sci 50: $1674-1680$.

Roff DA (1992). The Evolution of Life Histories: Theory and Analysis. Chapman \& Hall: London, UK.

Rollinson N, Hutchings JA (2013). Environmental quality predicts optimal egg size in the wild. Am Nat 182 (doi:0.1086/670648).

Räsänen K, Kruuk LEB (2007). Maternal effects and evolution at ecological time-scales. Funct Ecol 21: 408-421.

Sargent RC, Taylor PD, Gross MR (1987). Parental care and the evolution of egg size in fishes. Am Nat 129: 32-46.

Scott DP (1962). Effect of food quantity on fecundity of rainbow trout, Salmo gairdneri. J Fish Res Board Can 19: 715-731.

Solberg MF, Skaala O, Nilsen F, Glover KA (2013). Does domestication cause changes in growth reaction norms? A study of farmed, wild and hybrid atlantic salmon families exposed to environmental stress. PLoS One 8: e54469.

Sørensen MK, Norberg E, Pedersen J, Christensen LG (2008). Invited review: crossbreeding in dairy cattle: a Danish perspective. J Dairy Sci 91: 4116-4128.

Thorpe JE, Miles MS, Keay DS (1984). Developmental rate, fecundity and egg size in Atlantic salmon, Salmo salar L. Aquaculture 43: 289-305.

Utter F, Epifanio J (2002). Marine aquaculture: genetic potentialities and pitfalls. Rev Fish Biol Fish 12: 59-77.

Willham RL (1980). Problems in estimating maternal effects. Livest Prod Sci 7: 405-418. Wolf JB (2000). Gene interactions from maternal effects. Evolution 54: 1882-1898.

Wolf JB, Brodie ED III, Cheverud JM, Moore AJ, Wade MJ (1998). Evolutionary consequences of indirect genetic effects. Trends Ecol Evol 13: 64-69.

Wolf JB, Wade MJ (2009). What are maternal effects (and what are they not)? Philos Trans $R$ Soc Lond B Biol Sci 364: 1107-1115. 